\title{
Gestión de casos: adaptación para pacientes pluripa- tológicos y cuidadores en Colombia
}

\section{Case management: adaptation for patients with comorbidity and caregivers in Colombia Gestão de casos: adaptação de pacientes multimorbidos e cuidadores na Colômbia}

\author{
Sandra Milena Hernández-Zambrano ${ }^{1}$ \\ Sonia Patricia Carreño-Moreno ${ }^{2}$ \\ Ruth Alexandra Castiblanco-Montañez ${ }^{3}$ \\ Lorena Mesa-Melgarejo ${ }^{4}$ \\ Lorena Chaparro-Díaz \\ Ana Julia Carrillo-Algarra ${ }^{6}$ \\ Elena Gonzalo-Jiménez ${ }^{7}$
}

\section{Resumen}

Objetivo: Identificar puntos de consenso de tomadores de decisiones, profesionales, y usuarios, frente a aspectos fundamentales que debe contener un Modelo de Gestión de Casos en el contexto colombiano, para atender a personas en situación de pluripatología y sus cuidadores familiares. Método: Estudio descriptivo. Método de consenso entre expertos y muestreo por conveniencia. Se realizó la técnica Delphi de tiempo real en dos rondas. El análisis incluyó promedios aritméticos para determinar las propuestas más importantes y gobernables. $\boldsymbol{R} \boldsymbol{e}$ sultados: Participaron 25 expertos en la primera ronda y 23 en la segunda. Se obtuvo mayor consenso en: mayor disponibilidad de recursos económicos para garantizar infraestructura y recursos humanos suficientes; cualificación de recurso humano; potenciar la figura de la enfermera gestora de casos; implementación de rutas de atención integrales; sistemas de información que faciliten el tránsito y conexión de los usuarios; planes de alta vinculación de actores sociales para fomentar la autogestión. Conclusión: La adaptación del Modelo de GC en el sistema de aseguramiento colombiano, permitió identificar factores sustanciales para implementarlo en los niveles macro, meso y micro del sistema de salud, reconocido por los actores como altamente fragmentado.

Palabras clave: Gestión de casos, enfermería, multimorbilidad, enfermedad crónica, enfermería de práctica avanzada, cuidadores

\footnotetext{
Abstract

Objective: Identify the points of consensus of decision makers, professionals, and users, regarding the fundamental aspects a Case Management Model should have in the Colombian context, to care for people with comorbidity and their family caregivers. Method: Descriptive study. Consensus decision-making among experts and convenience sampling. The Delphi method was applied in real time for two rounds. The analysis included arithmetic means to determine the most important and manageable proposals. Results: 25 experts participated in the first round and 23 in the second round. A greater consensus was obtained in: availability of economic resources to guarantee sufficient infrastructure and human resources; qualification of human resources; potentiate the figure of the nurse in case management; implementation of comprehensive health care pathways; information systems that facilitates the transition and connection of the users; plans for high engagement of social actors to pro-
}

\begin{abstract}
Autor de correspondencia*
${ }^{1 *}$ Enfermera. PhD en Ciencias de la Salud. Mg en Cuidados de Salud para la Promoción de la Autonomía de las Personas y la Atención a los Procesos de Fin de Vida. Docente investigadora. Facultad de Enfermería. Fundación Universitaria Ciencias de la SaludFUCS. Bogotá, Colombia. Correo: smhernandez3@fucsalud.edu.co 0000-0001-5504-3065
\end{abstract}

${ }^{2}$ Enfermera. Mg en Enfermería énfasis en cuidado al paciente crónico. $\mathrm{PhD}$ en Enfermería. Profesora Asistente. Facultad de Enfermería. Universidad Nacional de Colombia. Bogotá, Colombia. Correo: spcarreno@unal.edu.co. (D) 0000-0002-4386-6053

${ }^{3}$ Enfermera. Magister en Salud Pública. Profesora asistente. Coordinadora de Investigaciones. Facultad de Enfermería. Grupo Perspectivas del Cuidado. Docente. Facultad de Enfermería. Fundación Universitaria de Ciencias de la Salud - FUCS. Bogotá, Colombia. Correo: racastiblanco@fucsalud.edu.co (D) 0000-0001-8328-9601

${ }^{4}$ Enfermera. Mg en Salud Pública y Mg en Economía de la Salud. PhD en Salud Pública. Líder. Unidad de métodos cualitativos e investigación social. Instituto de Evaluación Tecnológica en SaludIETS. Bogotá, Colombia. Correo: 1pmesam@unal.edu.co (D) 0000-00022781-080X

${ }^{5}$ Enfermera. Mg en Enfermería. PhD en Enfermería. Profesora Asistente. Facultad de Enfermería. Universidad Nacional de Colombia. Bogotá, Colombia. Correo: olchaparrod@unal.edu.co (D) 0000-0001-8241-8694

${ }^{6}$ Enfermera. Mg Administración en Salud. Facultad de Enfermería. Docente Fundación Universitaria Ciencias de la Salud- FUCS. Bogotá, Colombia. Correo: ajcarrillo@fucsalud.edu.co (D) $\underline{0000-0002-3765-7474}$ 


\section{CienciaenCuidado}

\section{Scientific Journal of Nursing}

mote self-management. Conclusion: The adaptation of the Case Management Model in the Colombian insurance system, allowed the identification of substantial factors to implement it in the macro, meso and micro levels of the health care system, recognized by the actors as highly fragmented.

Keywords: Case management, nursing, multy-morbidity, chronic disease, advanced nursing practices, caregivers.

\section{Resumo}

Objetivo: identificar os pontos em comum de tomadores de decisões, profissionais e usuários, perante aspectos fundamentais a considerar-se no Modelo de Gestão de Casos (MGC) no contexto colombiano, para atender as pessoas em situação de múltiplas patologias e os seus cuidadores familiares. Materiais e métodos: estudo descritivo. Método de consenso entre expertos e amostragem por conveniência. Realizou0-se a técnica Delphi de tempo real em duas chances. A análise incluiu medias aritméticas para determinar as propostas mais importantes e governáveis. Resultados: participaram 25 expertos na primeira virada e 23 na segunda. Obteve-se maior consenso em: maior disponibilidade de recursos financeiros para garantir a infraestrutura e recursos humanos suficientes; qualificação do recurso humano; potenciar a figura da enfermeira gestora de casos; implementação de rotas de atendimento integrais; sistemas de informação que facilitem a transição e conexão entre os usuários; planos de alta vinculação de atores sociais para o fomento da autogestão. Conclusão: a adaptação do MGC no sistema de saúde colombiano, permitiu identificar fatores substanciais para implementá-lo nos níveis macro, intermediários e micro do sistema de saúde, reconhecido pelos atores como altamente fragmentado.

Palavras-chave: enfermagem, multimorbidade, doença crônica, prática avançada de enfermagem, cuidadores.
7 Enfermera. Mg en Investigación en ciencias socio-sanitarias. Docente. Escuela Andaluza de Salud Pública. Granada, España. Correo: elena.gonzalo. easp@juntadeandalucia.es (i) $\underline{0000-}$ 0003-3815-6150
Recibido: 23 diciembre 2020 Aprobado: 25 abril 2021

Para citar este artículo / To reference this article / Para citar este artigo:

Hernández-Zambrano SM, Carreño-Moreno SP, Castiblanco-Montañez RA, Mesa-Melgarejo L, Chaparro-Díaz L, Carrillo-Algarra AJ, et at,. Gestión de Casos adaptación para pacientes pluripatológicos y cuidadores en Colombia. Rev. cienc. cuidad. 2021; 18(2):69-81. https://doi. org/10.22463/17949831.2815

(C) Universidad Francisco de Paula Santander. Este es un artículo bajo la licencia CC-BY-NC-ND

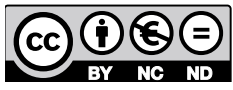

\section{Introducción}

El aumento sostenido de morbimortalidad por Enfermedades Crónicas no Transmisibles (ECNT), reta a profesionales, instituciones y estado, por su alta complejidad y costo. Mundialmente las ECNT significan una alta carga de la enfermedad y afectan a personas vulnerables y familiares, que socialmente son desfavorecidas, por exposición a factores de riesgo, acceso limitado a servicios sanitarios y costos desorbitados del prolongado tratamiento (1). Según la Organización Mundial de la Salud (OMS), el 71\% de muertes mundiales son causadas por ECNT, en millones de personas por año: 17,9 por enfermedades cardiovasculares; por cáncer 9,0 ; por enfermedades respiratorias 3,$9 ; \mathrm{y}$, por diabetes $1,6(1)$.

La ECNT, es una situación compleja poco explorada, descrita como multimorbilidad o pluripatología. Se caracteriza porque dos o más condiciones crónicas afectan a una persona, a diferencia de la comorbilidad, que hace referencia a enfermedades primarias que coexisten, pero no dependen una de la otra $(2,3)$. Esta condición implica fragilidad clínica, pérdida de autonomía y capacidad funcional, elevada utilización de recursos sociales, económicos y de salud; además, es de alto riesgo por sus complicaciones y mortalidad $(2,3)$. Las personas con ECNT, viven diferentes etapas: inicio y adaptación a la enfermedad; fase de progresión; y, la de mayor complejidad que aumenta las visitas a urgencias, con ingresos hospitalarios y complicaciones (4).

Colombia, respecto al impacto de la cronicidad compleja, conoce la alta carga en años de vida potencialmente perdidos (AVPP), años de vida vividos con discapacidad (AVD) y tasa de consulta por mil habitantes que oscila de 368 a $774(5,6)$. Un estudio sobre multimorbilidad devela que "afecta principalmente a las mujeres, se incrementa progresivamente con la edad, tiene alto impacto en los servicios de salud y en la calidad de vida de los pacientes; cerca de once millones de personas consultan cada año por patologías crónicas, y casi la mitad presenta multimorbilidad" (7). A esta problemática se añade la atención fragmentada, desintegrada, desconectada y costosa del sistema de salud (8). 


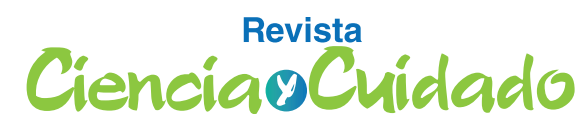

Scientific Journal of Nursing
Sandra Milena Hernández-Zambrano, Sonia Patricia Carreño-Moreno, Ruth Alexandra Castiblanco-Montañez, Lorena Mesa-Melgarejo, Lorena Chaparro-Díaz, Ana Julia Carrillo-Algarra, Elena Gonzalo-Jiménez
Por otra parte, la ECNT impacta negativamente los ámbitos epidemiológico y económico; además, en las personas y familias deteriora su calidad de vida, aumenta el gasto, las aísla socialmente, disminuye el bienestar espiritual y aumenta la carga del cuidado (8-13). Lo planteado anteriormente, hace indispensable reorientar el modelo de atención colombiano, hacia uno que responda a la complejidad y necesidad de integración, conexión, coordinación y continuidad y lo que implica su abordaje. Numerosos sistemas sanitarios comparten el reto de adecuar sus estructuras y modelos asistenciales al aumento de las ECNT para garantizar la continuidad asistencial a personas que requieren diferentes profesionales; así como a servicios y tecnologías localizados en distintas organizaciones e incluso en más de un sector.

En el marco de los modelos de atención a personas con condiciones crónicas, como el Innovative Care for Chronic Conditions de la OMS, con la gestión de casos (GC) es una de las estrategias adoptadas por los mejores sistemas sanitarios de países de Norte América y Europa, porque integra las intervenciones de diferentes servicios y profesionales, en torno a pacientes con necesidades de salud complejas. Es por eso, que para lograr resultados costo-efectivos despliega acciones como: captar los pacientes; valorar cada caso con enfoque integral y multidimensional; diseñar un plan asistencial adecuado que implique al paciente y al conjunto de profesionales para la solución de sus problemas; coordinar intervenciones en torno al paciente, modificando el plan asistencial conforme evolucione la situación; $y$, facilitar el acceso a recursos socio-sanitarios o ayudas técnicas de apoyo al cuidado.

Otro rasgo a tener en cuenta es que el cuidado familiar es de interés en los programas de GC, para fortalecerlo, complementarlo o sustituirlo, protegiendo y apoyando a quienes lo brindan. En resumen, para la población con ECNT complejas y, o pluripatología, la GC es una alternativa asistencial orientada a satisfacer las necesidades del paciente, involucrándolo junto al cuidador familiar, en la toma de decisiones y las intervenciones. La mayoría de los programas de gestión de casos documentados en la literatura están liderados por Enfermeras de Práctica Avanzada (EPA) $(14,15)$.

Con respecto a la evidencia científica sobre los efectos de la GC se destacan: mejoras de la calidad de vida; aumento de la capacidad funcional; reducción de caídas, en personas mayores que viven en su domicilio; mejora en los resultados clínicos; disminución de complicaciones; aumento de satisfacción en pacientes y cuidadores; disminución de costos financieros en salud derivados de reducción de polifarmacia, de ingreso a instituciones de cuidado a largo plazo y hospitalizaciones; y, días de estancia y consultas de salud, en diferentes poblaciones (16-18).

En Colombia, existe interés por mejorar la atención de las personas con ECNT múltiples, escenario propicio para definir políticas socio sanitarias, de Talento $\mathrm{Hu}-$ mano en Salud, y modelos de atención. En este contexto, la Gestión de Casos es un modelo de provisión de servicios de salud efectivo y apropiado para que las aseguradoras garanticen atención integral y continuada (19). Por tanto, el objetivo de este estudio es identificar puntos de consenso de tomadores de decisiones, profesionales y usuarios, frente a aspectos fundamentales que deben contener un modelo de GC en el contexto colombiano, para la atención de personas en situación de pluripatología y sus cuidadores familiares.

\section{Métodos}

\section{Diseño y población}

Este es un estudio descriptivo, utilizando el método de consenso entre expertos. Los participantes se segmentaron en tres grupos: a) Usuarios, entendidos como personas en condición de cronicidad, sus cuidadores familiares, y líderes de asociaciones de pacientes; b) Profesionales de salud, provenientes de atención primaria y especializada; y c) Tomadores de decisiones del ámbito de la macro y meso gestión, provenientes del Ministerio de Salud y Protección Social, Secretaría de Salud, Empresas Promotoras de Salud (EPS) que son las aseguradoras e Instituciones Prestadoras de Servicios de Salud (IPS). El muestreo se realizó por conveniencia, con los siguientes criterios de inclusión: residir en Bogotá (Colombia) y contar con experiencia vivencial o profesional mayor a 3 años en el ámbito de la cronicidad.

\section{Recolección de datos}

Se realizó la técnica Delphi en dos rondas, entre abril y mayo del 2018, utilizando la variante de tiempo real que, según Varela Ruiz et al., (20) "se caracteriza por ser una variante más corta porque el proceso se lleva a cabo durante una reunión a través de mecanismos para resumir las respuestas dadas inmediatamente". En la fase de preparación, el equipo investigador identificó aspectos fundamentales para el consenso mediante la revisión de literatura y normatividad vigente. Estos aspectos se registraron en una matriz $5 \mathrm{~W} 2 \mathrm{H}$, que se utilizó en las fases siguientes para consensuar las propuestas 
de adaptación del modelo de GC al sistema de aseguramiento colombiano. Las cinco situaciones problema fueron: restricciones de acceso, barreras de oportunidad y administrativas, fragmentación de la atención y fallas de comunicación en el sistema de salud.

Ronda 1. Identificación y consenso de componentes, competencias, acciones y actores para la adaptación del modelo de gestión de casos. Para conformar el panel de expertos se enviaron cartas de invitación a 60 candidatos. Respondieron 25 quienes conformaron el panel de expertos inicial. Se conformaron 3 grupos para realizar reuniones separadas con usuarios, profesionales de salud y tomadores de decisión, con el objetivo de desarrollar y consensuar la matriz de propuestas. Para cada reunión, los participantes mantuvieron el anonimato mediante el uso de nombres ficticios. Las reuniones comenzaron con la presentación del Modelo de Gestión de Casos, durante 20 minutos, de la que se entregó una síntesis a los participantes A continuación, se conformaron grupos de discusión para diligenciar la matriz. Cada grupo de discusión duró entre 60 y 90 minutos y fue grabado en audio, previo consentimiento de los integrantes. Los datos fueron transcritos y analizados a partir de las 5 dimensiones predefinidas en la matriz y se sometieron a un análisis temático, que posibilitó identificar la perspectiva de consenso de los participantes acerca de las propuestas de adaptación del modelo de gestión de casos.

Ronda 2. Priorización de las propuestas según su Importancia y Gobernabilidad. Consistió en una ronda anónima de priorización a través de un formulario electrónico creado en Google Forms. Cada grupo escuchó las propuestas consensuadas en la primera ronda, y mediante un dispositivo electrónico, las calificó, en una escala de cero a cinco, por nivel de Importancia, entendida como un aspecto clave e indispensable para el desarrollo y obtención de resultados del modelo de gestión de casos; y la Gobernabilidad, definida como la capacidad y control que se tiene sobre el aspecto a priorizar.

\section{Análisis de datos}

Los resultados de la priorización se analizaron con base en los promedios aritméticos de las respuestas y se ubicó cada una de las propuestas en un plano cartesiano, para seleccionar la que se ubicaba más al extremo superior derecho, por ser la más importante y gobernable. Los resultados se socializaron y fueron validados por los participantes.

\section{Aspectos éticos}

Este estudio se ajustó a normas internacionales para investigación con seres humanos. La participación fue voluntaria, se hizo el proceso de consentimiento informado del cual se obtuvo documento firmado. Se contó con autorización escrita para la grabación. El estudio tiene el aval del Comité de Ética en Investigación en Seres Humanos (CEISH) del Hospital de San José (acta 9 del 24 de mayo de 2017).

\section{Resultados}

\section{Características de los participantes}

De los 60 expertos, invitados a formar parte del panel, participaron 25 en la primera ronda y 23 en la segunda (6 hombres y 17 mujeres), que se distribuyeron así: usuarios $(n=4)$, profesionales $(n=8)$ y tomadores de decisiones $(n=8)$.

Por otra parte, el grupo de tomadores de decisiones conformado por 8 profesionales, del cual el $87,5 \%$ eran mujeres, edad promedio 41.9 años, siendo: 6 Enfermeras(os), 3 Médicos(as) y 1 Odontólogo(a). Con relación al grupo de profesionales participaron: 4 mujeres y 4 hombres, 3 médicos, 3 enfermeras, 1 psicólogo, una trabajadora social; con edad promedio de 40.5 años. El grupo de usuarios lo conformaron 6 mujeres y 1 hombre, edad promedio 51 años. La distribución fue homogénea: cuidadores $(n=2)$, pacientes $(n=3)$ y líderes de usuarios $(n=2)$.

\section{Análisis cualitativo}

En cada grupo de participantes, el análisis de las posibles soluciones de las problemáticas del sistema se planteó teniendo en cuenta las potencialidades que el modelo de $\mathrm{GC}$ ha mostrado en otros contextos y su adaptación al sistema de salud colombiano, desde sus conocimientos y experiencias cotidianas. La Tabla 1 presenta fragmentos originales de discursos significativos de los participantes.

Para los tomadores de decisiones, la política de atención integral proporciona el marco normativo y teórico necesario para adaptar el modelo al sistema de salud del país, ya que por un lado, el enfoque de gestión de riesgo permite conciliar el modelo y el sistema; y, por otro, las Rutas integradas de atención en salud (RIAS) descritas en la política sustentan estos modelos de atención a pacientes. Hay que precisar que su implementación facilitaría el desarrollo de un modelo como el de GC y resolvería problemas como las barreras de acceso y fragmentación de la atención. La mayoría de 


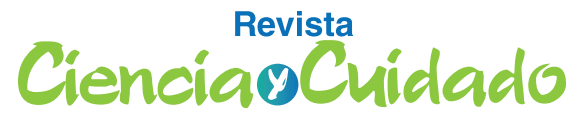

Scientific Journal of Nursing los tomadores de decisión consideró que están dadas las condiciones para implementarlo; también, emerge repetidamente que la clave operativa está en implementar un sistema de información compartido por los diversos actores, permitiendo conocer la situación de salud del paciente a todo nivel, incluido el domiciliario. Otro tema de discusión, son los mecanismos de contratación entre asegurador y prestador de servicios, como elemento que facilita el adecuado despliegue de los atributos del modelo.

De igual manera, los profesionales de salud consideran vital, para adaptar el modelo al sistema colombiano, desarrollar e implementar sistemas de información actualizados que permitan una mejor coordinación entre los actores, y que sirvan para erradicar trámites innecesarios para el paciente; además, evidencian la necesidad de contar con mayor recurso humano para lograr la atención oportuna e integral que implica el modelo. Del mismo modo, consideran que para el sistema colombiano, el rol de la GC debe centrarse en favorecer la articulación entre niveles, y la coordinación entre profesionales. El grupo reitera que los trámites administrativos para autorizar servicios, se constituyen en la principal falla del sistema, afectando la atención integral y la oportunidad. Para solucionarla, proponen un sistema de información que incorpore la autorización directa, cuando el profesional solicite los servicios para el paciente.

Con respecto a los usuarios la distancia entre el derecho a la salud, los aspectos normativos y cómo opera realmente el sistema son las principales problemáticas a resolver para implementar el modelo, así como la pérdida del sentido de lo que es el Ser Humano al interior del sistema. Igualmente, hay que adaptar el modelo, para lo cual se requiere una mejor infraestructura y acceso a las estrategias de promoción y prevención para evitar complejizar situaciones de salud. Simultáneamente, que hay que favorecer la integralidad y seguimiento en el hogar que posibilite gestionar realmente el caso y articular el equipo interdisciplinario, son atributos del modelo de gestión de casos. Para este grupo, reconocer la labor del cuidador y que exista una mayor integración de la familia, son aspectos relevantes para posicionar el modelo y aceptarlo; asimismo, georreferenciar el cuidado puede significar innovación y aporte para los aseguradores y el sistema, facilitando la disposición de los recursos.

Hay que indicar que como aspectos ausentes para una adecuada adaptación del modelo de gestión de casos, al sistema de salud colombiano emergen: la falta de adecuación a los territorios rurales, en especial a los dispersos, y la no articulación con políticas de talento humano en salud.

Tabla 1. Verbatim relevantes de los participantes

\begin{tabular}{|c|c|}
\hline Grupo & Verbatim relevantes \\
\hline $\begin{array}{l}\text { Tomadores } \\
\text { de decisiones }\end{array}$ & $\begin{array}{l}\text { T1. "¿Adaptando las rutas integrales de atención? adaptando, adoptando e implementando". } \\
\text { T2. "Un atributo importante es la orientación a resultados, porque compromete alprofesional respons- } \\
\text { able de la gestión del caso a velar porque se cumplan los objetivos del plan asistencial y alimentar el } \\
\text { sistema de información para que permita evaluar resultados e identificar oportunidades de mejora”. } \\
\text { T3. "Creo que el atributo es la continuidad, es lo que pretende el enfoque de re- } \\
\text { des integrales y las rutas de atención (...), se espera que el modelo integral las ru- } \\
\text { tas de atención solucionen en gran medida las barreras en autorizaciones. } \\
\text { T4. "Por eso salieron las rutas integrales de atención (RIAS), que están tratando de integrar y bus- } \\
\text { car accesos a la ruralidad, que es donde tenemos esos problemas porque las personas, tienen co- } \\
\text { bertura, pero no acceso, están afiliados a una EPS, pero no ingresan al sistema. La idea es, hacerlo } \\
\text { integral con las RIAS, es lo que está sacando el ministerio ahora y estamos las EPS trabajando". } \\
\text { T5. "No basta el sistema de información integral, tiene que haber una oper- } \\
\text { atividad de las rutas y el modelo en si y la articulación de todas las redes". } \\
\text { T6. "Tiene que ver con el sistema de información, porque si tuviéramos un sistema integral, donde tu- } \\
\text { viéramos acceso a todas las atenciones que ha tenido el paciente y a todas sus comorbilidades, se po- } \\
\text { dría redireccionar dentro de los integrantes del equipo de saludpara cada necesidad que se detecte". }\end{array}$ \\
\hline
\end{tabular}


Scientific Journal of Nursing

Profesionales P1. "No hay coordinación asistencial entre atención primaria y especializada, ni entre los profesionales que atienden a la persona, sistemas de información fragmentados". P2. “(...) Fallas en la comunicación dentro del sistema, no hay plan de egreso que detalle la información del paciente que va al domicilio, no hay una historia clínica única”. P3. "No hay disponibilidad del recurso para atender la demanda, les mandan algo y van a pedir la cita y no hay, les toca esperar o nunca se las dan". P4. "Si no hay agenda, es porque no hay recursos para que abran otro consultorio y paguen otro médico, en mi realidad, veo es que no hay recursos". P5. "Tiene que haber una relación directa entre la gestora de casos y los entes administrativos (...), y conexión entre la EPS y la gestora, a la cual se le autoriza”. P7. "Creo que el tema de las autorizaciones debería ser eliminado, si la EPS tiene relación directa con su IPS, usted va el médico le pide algo y lo pide de una, no debería ir hasta la EPS que lo autoricen y volver otra vez".

Usuarios U1. "La sensación molesta de uno cuando pasa por un servicio y se encuentra con esa restricción de acceso, en los casos que hemos tenido pacientes crónicos donde está en juego la vida, la persona, es que como que al sistema le interesa es que se muera rápido la persona, no tratarla, rehabilitarla y mantenerle su calidad de vida hasta el final, sino entre más rápido se muera es mejor." (...) "Cuando mencionabas lo de infraestructura, ahi también está la adecuación de espacios propicios para mejorar la prestación, bueno y pues que el servicio tenga mayor acceso, no sé si ahi también entren como de manera conjugada". U2. "Pensaría que, por el lado del sistema, una buena red de comunicación, para que no tenga precisamente lo que comentaba la jefa $\mathrm{H}$, en la parte administrativa, ¿cómo esas autorizaciones que se presentan en esos problemas no?, mayor comunicación entre las EPS y las IPS para que se pueda descongestionar la parte administrativa (...) es muy dificil que las IPS tengan acceso a las historias o al manejo de las EPS, tendría que hacerse un vínculo". C2. "Al igual que los profesionales de la salud, la enfermera lo atiende a uno de día y de noche, o por turnos, y tiene razón, yo entiendo eso, pero los cuidadores no tienen tiempo, límite ni de día ni de año, ni lunes, ni días de fiesta (...), va a llegar un momento en que el sistema laboral tiene que tenerlos en cuenta, porque no es justo, uno comprende el sentimiento, la parte familiar, todo eso, pero eso le corresponde es al sistema (...) y generalmente, el rol del cuidador en nuestra sociedad está en manos de la mujer y más de la que no se casó, es otra injusticia, como no formaron familia, tienen que estar a cargo de la mamá, o el papá". C1. "La atención de salud que tenemos en Bogotá es de mejor calidad que la de Leticia, allí puede que la EPS quiera otorgar los medicamentos, perono han llegado(...), esono tiene sentido, si la persona ya tiene diagnosticada enfermedad, tratamiento, medicamentos, debe ser claro que los reciba esté donde esté". C1. "La persona que padece una enfermedad crónica debe vivir hasta el final, no sobrevivir sino vivir, tener la oportunidad de vivir el derecho, la dignidad". C1. "El funcionario debe ser engranado, el modelo me parece excelente, y mejor que el de cuidados paliativos, he trabajado en cuidados paliativos porque los médicos ante una enfermedad crónica que la persona no va a recuperar la salud, por el contrario cada día la va a perder más, si no pueden hacer nada, es mucho más viable que existiera este modelo, me parece genial, es que a la enfermera se le ha recluido en un sistema asistencial, y ella tiene una capacidad de gestión impresionante, comprende muchas cosas mejor que los mismos especialistas".

Fuente: Elaborado por las investigadoras: *Tomadores de decisiones (T), Profesionales (P), Pacientes (U)

y Cuidadores (C).

\section{Priorización de propuestas según situación problema}

En la primera ronda con los 3 grupos se generaron 50 propuestas; los tomadores de decisiones postularon el $40 \%$, el grupo de profesionales el $30 \%$ y los usuarios el $30 \%$. Fueron calificadas según Importancia y Gobernabilidad, se priorizó la propuesta con mayor promedio. A continuación, se describen las propuestas priorizadas por cada grupo de expertos en las 5 situaciones problema analizadas: restricciones de acceso, barreras de oportunidad y administrativas, fragmentación de la atención y fallas en procesos de comunicación dentro del sistema de salud.

Situación problema: restricciones de acceso
Para el grupo de tomadores de decisión, primó la implementación de las RIAS, así como asegurar la red de prestación de servicios, que debe ser liderada por la EPS para: disminuir los costos, garantizar oportunidades, continuar la atención y eliminar barreras. Incluye la caracterización de la población con un enfoque de gestión del riesgo y contratación adecuada de servicios de acuerdo con el diagnóstico de la población. En cuanto a los profesionales de salud se priorizó la coordinación de la atención, la autogestión, un sistema de información actualizado, personal cualificado y capacitación a los usuarios en procesos internos de las instituciones. Al respecto, la responsabilidad recae sobre las IPS, de manera anticipada o simultánea cuando el paciente lo requiera, dado que las barreras se pre- 
CienciäoCuidado

Scientific Journal of Nursing sentan en las instituciones, impidiendo que se gestione un modelo centrado en el paciente y sus necesidades reales.

Los usuarios, por su parte, plantean aumentar la asignación de personal médico especialista, infraestructura, el tiempo para la prestación de los servicios y agilizar los procesos entre IPS y EPS. Para ello es necesario aumentar recursos físicos y económicos con el objeto de ampliar horarios de atención entre semana y fines de semana; además, se debe aumentar el presupuesto en programas de prevención, que es lo que más aqueja a los usuarios (Ver Tabla 2).

Tabla 2. Propuestas priorizadas en situación problema: restricciones de acceso, barreras de oportunidad y barreras administrativas

\begin{tabular}{|c|c|c|c|c|}
\hline $\begin{array}{l}\text { Situación } \\
\text { problema }\end{array}$ & $\begin{array}{c}\text { Propuesta prior- } \\
\text { izada }\end{array}$ & $\begin{array}{c}\text { Pro- } \\
\text { medio }\end{array}$ & $\begin{array}{l}\text { Grupo que pri- } \\
\text { orizó }\end{array}$ & ¿En qué consiste? \\
\hline \multirow[t]{3}{*}{$\begin{array}{l}\text { Restricciones } \\
\text { de acceso }\end{array}$} & $\begin{array}{l}\text { Propuesta C. } \\
\text { Implementación de } \\
\text { RIAS }\end{array}$ & 4,0 & $\begin{array}{l}\text { Tomadores de } \\
\text { decisión }\end{array}$ & $\begin{array}{l}\text { Implementar rutas de atención y } \\
\text { garantizar la red de prestación de } \\
\text { servicios. }\end{array}$ \\
\hline & $\begin{array}{l}\text { Propuesta } \mathrm{C} \text {. } \\
\text { Coordinación de la } \\
\text { atención }\end{array}$ & 4,2 & $\begin{array}{l}\text { El profesionales } \\
\text { de salud }\end{array}$ & $\begin{array}{c}\text { Coordinación de la atención, auto- } \\
\text { gestión y sistema de información } \\
\text { actualizado. }\end{array}$ \\
\hline & $\begin{array}{l}\text { Propuesta B. Mayor } \\
\text { número de talento } \\
\text { humano en salud }\end{array}$ & 4,2 & Usuarios & $\begin{array}{l}\text { Asignación de mayor personal médi- } \\
\text { co especialista. }\end{array}$ \\
\hline \multirow[t]{3}{*}{$\begin{array}{l}\text { Barreras de } \\
\text { oportunidad }\end{array}$} & $\begin{array}{l}\text { Propuesta A. } \\
\text { Articulación de } \\
\text { EAPB e IPS }\end{array}$ & 4,1 & $\begin{array}{l}\text { Tomadores de } \\
\text { decisión }\end{array}$ & $\begin{array}{l}\text { Articulación de los atributos de cal- } \\
\text { idad, para mitigar la situación prob- } \\
\text { lema }\end{array}$ \\
\hline & $\begin{array}{l}\text { Propuesta A. } \\
\text { Gestión de los recur- } \\
\text { sos }\end{array}$ & 4,3 & $\begin{array}{l}\text { El profesionales } \\
\text { de salud }\end{array}$ & $\begin{array}{l}\text { Gestionar recursos económicos y } \\
\text { humanos que permitan aumentar la } \\
\text { disponibilidad y el acceso. }\end{array}$ \\
\hline & $\begin{array}{l}\text { Propuesta B. Aumen- } \\
\text { to de especialistas }\end{array}$ & 4,6 & Usuarios & $\begin{array}{l}\text { Promover la preparación y con- } \\
\text { tratación de especialistas en el país. }\end{array}$ \\
\hline \multirow[t]{3}{*}{$\begin{array}{l}\text { Barreras ad- } \\
\text { ministrativas }\end{array}$} & $\begin{array}{l}\text { Propuesta C. } \\
\text { Tecnologías para evi- } \\
\text { tar suplantaciones }\end{array}$ & 3,8 & $\begin{array}{l}\text { Tomadores de } \\
\text { decisiones }\end{array}$ & $\begin{array}{l}\text { Implementación de tecnología para } \\
\text { evitar suplantaciones. }\end{array}$ \\
\hline & $\begin{array}{l}\text { Propuesta A. } \\
\text { Eliminación de las } \\
\text { barreras }\end{array}$ & 4,1 & $\begin{array}{l}\text { El profesionales } \\
\text { de salud }\end{array}$ & $\begin{array}{c}\text { Eliminar las barreras de acceso a los } \\
\text { servicios de salud. }\end{array}$ \\
\hline & $\begin{array}{l}\text { Propuesta B. } \\
\text { Unificación y virtual- } \\
\text { ización de trámites }\end{array}$ & 4,9 & Usuarios & $\begin{array}{l}\text { Centralización del lugar donde se } \\
\text { realizan los trámites administrativos. }\end{array}$ \\
\hline
\end{tabular}

Fuente: Elaborado por las investigadoras.

\section{Situación problema: barreras de oportunidad}

Los tomadores de decisiones plantearon en su propuesta darle prioridad a la articulación de los atributos del sistema de salud, para realizar una reestructuración organizacional, basada en el diagnóstico de necesidades de los usuarios. En este sentido, hay que privilegiar un enfoque de gestión de riesgo, para adecuar la capacidad instalada de las IPS, con el fin de eliminar barreras para satisfacer las necesidades en salud de la población. Los profesionales de salud, por su parte, señalaron la importancia de la gestión de recursos económicos y humanos que permitan disponibilidad y acceso a los servicios integrales de salud. En este sentido, es necesario aumentar el recurso humano especializado, incluyendo el rol de la Gestora de casos. Este elemento debe ser 
gestionado por las aseguradoras, dado que es de vital importancia para el sistema de salud colombiano, articular todos los actores en los diferentes niveles de atención para lograr una comunicación directa y efectiva y así garantizar los derechos de los pacientes. Así mismo, los usuarios coinciden en la necesidad de mejorar la formación del recurso humano, especialmente los especialistas, y aumentar su remuneración, ya que las consultas con estos profesionales son las de mayor demanda y menor oferta; por tanto, la responsabilidad de esta actividad debe ser del Estado (Ver Tabla 2).

\section{Situación problema: barreras administrativas}

En la Tabla 2, se evidencia que los tomadores de decisiones priorizaron la implementación de tecnologías para evitar suplantaciones de identidad, a través de un sistema de información avanzado, que disminuya los costos y trámites, mejorando la calidad de la atención. Los profesionales de salud proponen la eliminación de barreras de acceso a los servicios de salud; proposición que se traduce en la agilización en el proceso de autorizaciones en las IPS, quienes deben contar con una comunicación directa con las EPS, ya que la variación en la contratación de la red de prestadores, se traduce en barreras para los pacientes. Lo anterior se puede ejecutar a través de canales electrónicos que agilicen los procesos de atención. Los usuarios concuerdan en que se debe centralizar en un lugar, en donde se realicen los trámites y procedimientos; también, plantean la tecnifi- cación de varios procesos y el uso de tecnologías de información, lo que disminuiría las barreras administrativas.

\section{Situación problema: fragmentación en la atención}

Para eliminar la fragmentación en la atención, los tomadores de decisiones proponen fortalecer el proceso de referencia y contra referencia por medio de un sistema de información implantado por las aseguradoras a nivel nacional, con el fin de evitar la vulneración del derecho a la salud que se refleja en la discontinuidad y en la poca oportunidad de atención integral en salud. Por consiguiente, se debe realizar una distribución adecuada de recursos físicos, económicos y talento humano en salud en todo el territorio nacional. Por otro lado, los profesionales aconsejan la creación de una red articulada de información, similar a los propuesto por los decisores en salud, donde participen todos los actores del sistema general de seguridad social. Propuesta que postulan con el objetivo de garantizar continuidad y acceso a la atención en salud; también, incluyen la necesidad de acceso a la historia clínica digital en todos los niveles de atención. Al respecto los usuarios proponen, la creación de gestores en salud disponibles en cada centro de salud, para educar a los pacientes en cuanto a trámites administrativos; asimismo crear más asociaciones de usuarios que propendan por la autogestión de sus procesos de enfermedad y mejoren su participación social (Ver Tabla 3).

Tabla 3. Propuestas priorizadas en situación problema: fragmentación de la atención y fallas en procesos de comunicación del sistema

\begin{tabular}{|c|c|c|c|c|}
\hline $\begin{array}{l}\text { Situación prob- } \\
\text { lema }\end{array}$ & Propuesta priorizada & Promedio & $\begin{array}{l}\text { Grupo que } \\
\text { priorizó }\end{array}$ & ¿En qué consiste? \\
\hline \multirow[t]{3}{*}{$\begin{array}{l}\text { Fragmentación } \\
\text { en la atención }\end{array}$} & $\begin{array}{l}\text { Propuesta D. } \\
\text { Fortalecimiento del proce- } \\
\text { so de referencia y contrar- } \\
\text { referencia }\end{array}$ & 4,4 & $\begin{array}{l}\text { Tomadores de } \\
\text { decisión }\end{array}$ & $\begin{array}{l}\text { Fortalecer el proceso de refer- } \\
\text { encia y contrarreferencia por } \\
\text { medio de un sistema de infor- } \\
\text { mación. }\end{array}$ \\
\hline & $\begin{array}{c}\text { Propuesta A. } \\
\text { Redes de información para } \\
\text { pacientes }\end{array}$ & 4,1 & $\begin{array}{l}\text { Profesionales } \\
\text { de salud }\end{array}$ & $\begin{array}{l}\text { Creación de redes articulados } \\
\text { de información del paciente. }\end{array}$ \\
\hline & $\begin{array}{c}\text { Propuesta A. Asociación } \\
\text { de usuarios autogestores } \\
\text { de la salud }\end{array}$ & 4,8 & Usuarios & $\begin{array}{l}\text { Creación de un grupo de ge- } \\
\text { stores en salud especializados } \\
\text { en trámites administrativos. }\end{array}$ \\
\hline
\end{tabular}




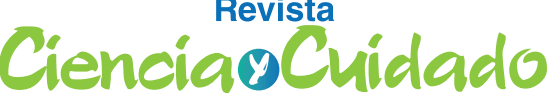

Scientific Journal of Nursing

\begin{tabular}{|c|c|c|c|c|}
\hline \multirow{3}{*}{$\begin{array}{l}\text { Fallas en pro- } \\
\text { cesos de comuni- } \\
\text { cación del siste- } \\
\text { ma }\end{array}$} & \multirow{3}{*}{$\begin{array}{l}\mathrm{Si} \\
\mathrm{P}\end{array}$} & 4,4 & $\begin{array}{l}\text { Tomadores de } \\
\text { decisiones }\end{array}$ & $\begin{array}{c}\text { Sistema de información único, } \\
\text { historia clínica única e inte- } \\
\text { grada }\end{array}$ \\
\hline & & 4,2 & $\begin{array}{c}\text { Profesionales } \\
\text { de salud }\end{array}$ & $\begin{array}{l}\text { Articular la información entre } \\
\text { todos los actores que participan } \\
\text { dentro del sistema de salud. }\end{array}$ \\
\hline & & 4,7 & Usuarios & $\begin{array}{l}\text { Formalización del protocolo } \\
\text { del egreso del paciente. }\end{array}$ \\
\hline
\end{tabular}

Fuente: Elaborado por las investigadoras.

Situación problema: fallas en procesos de comunicación del sistema

Para las fallas en procesos de comunicación, los tomadores de decisiones proponen un sistema de historia clínica unificada y digital en todas las aseguradoras del territorio nacional y sus instituciones afiliadas; esto con el fin de integrar todos los sistemas de información y garantizar el acceso a la información y el derecho a la salud en toda la nación. Los profesionales de salud dan prioridad a la integración de información en salud de todos los actores del sistema de salud nacional, incluido el Ministerio de Salud, para crear bases de datos unificadas que permitan atención continua, en cualquier momento y lugar del territorio nacional. A su vez, los usuarios desde su perspectiva como sujetos de cuidado plantean la estandarización del plan de egreso hospitalario, donde se integre al cuidador familiar con el equipo interprofesional, para agilizar el egreso y disminuir complicaciones o consultas. Es decir, que es necesario garantizar una comunicación efectiva entre el paciente, la familia y el equipo de salud antes de que el paciente salga de la institución (Ver Tabla 3).

\section{Discusión}

La adaptación del Modelo de GC en el sistema de aseguramiento colombiano, permitió identificar factores sustanciales para su implementación. Como elementos de mayor consenso identificados por los diferentes actores del sistema que participaron en el estudio, se evidencian: mayor disponibilidad de recursos económicos para garantizar la infraestructura y los recursos humanos suficientes; cualificación del recurso humano; potenciar la figura de la enfermera gestora de casos, como recurso costo efectivo para el sistema de salud; implementar rutas de atención integrales; promover sistemas de información que faciliten el tránsito y conexión de los usuarios entre los prestadores y aseguradores, profesionales y niveles de atención $(21,22)$; facilitar los planes de egreso hospitalarios y vincularlos con los ac- tores sociales para fomentar la autogestión. Al respecto, Morilla et al., (23) describen cómo estos factores deben fomentarse con la coordinación entre diferentes actores por niveles desde la macro, meso y micro gestión del sistema de atención; la primera, a través de la integración del plan de salud, la atención primaria y la atención especializada para evitar la condición de fragmentación en la atención; en la meso gestión, hay que fomentar los enlaces entre las instituciones prestadoras de servicios hospitalarios y los sociales para garantizar continuidad y atención integral a los sujetos de cuidado; y, en lo micro se deberá propiciar la autogestión en la diada paciente-cuidador.

Según los expertos, la GC en pacientes con alta complejidad permite mejorar la utilización de servicios de salud, al regular la demanda en los pacientes que tienen exceso de servicios o atención deficiente, facilitando el acceso a la atención al reducir trámites administrativos. Se han reportado efectos protectores de la GC sobre variables como reducción de visitas a urgencias, hospitalizaciones, reingresos y tiempo de estancia hospitalaria, deterioro funcional en el área hospitalaria y menor consumo de farmacia; lo que facilita la reducción en el consumo y costos de recursos hospitalarios, permitiendo un incremento en la atención primaria por el seguimiento y monitoreo domiciliario (24-29).

Así mismo, cuando se incluye la GC en los programas de egreso hospitalario, los beneficios se extienden al cuidador, puesto que es una estrategia que trasciende el cuidado de la díada persona con enfermedad crónica-cuidador familiar y el hogar. Algunos estudios reportan aumento en la competencia de cuidado, tanto en pacientes como en cuidadores y una reducción del $48 \%$ al 27\% de sobrecarga en los cuidadores, después de la intervención con la gestora de casos, porque se permiten espacios de detección y acción oportuna (30).

En este sentido, centrar la atención en el sujeto de cuidado permite que los profesionales tengan mayor con- 
ciencia de las necesidades de su entorno, barreras y facilitar la adopción de servicios de apoyo, así como de los recursos disponibles para mejorar la autogestión y el abordaje del riesgo social (31). Además, la atención domiciliaria permite construir una relación más cercana con la diada de cuidado y comprender las experiencias de enfermarse, cuidarse y curarse, al incorporar las perspectivas de los diferentes actores. Al priorizar los factores anteriores se puede avanzar hacia una atención integral, transversal, de calidad y sostenible para las personas y sus cuidadores (32).

Dado que las enfermeras han adoptado el rol de gestoras dentro del modelo, actuando como principales profesionales de enlace y coordinación entre niveles a nivel mundial, se han instaurado nuevos perfiles de formación como el de Enfermeras de Práctica Avanzada, que cuenta con un mejor conocimiento del sistema, capacidad de resolución interacción interprofesional y de ajuste de las condiciones para la provisión de servicios adaptados a las necesidades de los pacientes crónicos (33). Por lo que se sugiere que las enfermeras cuenten con la capacitación en gestión de casos y experiencia profesional mínima de 3 años, para desempeñarse en este campo de acción. Se debe agregar, que las gestoras han sido descritas como un recurso costo efectivo para los sistemas de salud (31), ya que lideran al grupo de personal especializado y propician los recursos necesarios para atender las necesidades de acuerdo al nivel de complejidad del sujeto con pluripatología; por tanto, la gestión de casos es efectiva cuando la atención es suministrada por un equipo multidisciplinario y existe articulación entre proveedores de servicios sociales y de salud, que permitan optimizar los recursos personales y desarrollar una atención integral (34).

No obstante, diferentes expertos (18) proponen establecer un marco conceptual y legal de las competencias y funciones de la gestora sobre la implementación del modelo, gestión de recursos y atención de pacientes, dado que esta falencia ha generado ambigüedades y conflictos que interfieren en el éxito de la gestión de casos. A nivel nacional no se ha desarrollado aún normativa alguna que dé estabilidad a este nuevo perfil del enfermero, al ser un modelo que se encuentra en fase de evaluación.

Asimismo, se requieren mecanismos válidos para la detección de casos y ratios adecuados para la asignación de profesionales y especialistas; así como sistemas de registro accesibles y que se puedan compartir con los miembros del equipo o para la integración de servicios de salud y sociales (34). Al respecto el informe de distribución y oferta de especialistas a nivel nacional demuestra un déficit de profesionales que atiendan de manera oportuna a la población en condición de cronicidad, principalmente en regiones rurales (34). Por ello, se debe contar con una mayor disponibilidad de recursos económicos, a fin de garantizar la infraestructura y los recursos humanos suficientes para responder a las necesidades de la población, como lo sugieren los participantes en este proceso de adaptación del modelo de GC al contexto colombiano.

\section{Conclusiones}

- La adaptación del Modelo de GC en el sistema de aseguramiento colombiano, permitió identificar factores de la macro, meso y micro gestión que son sustanciales para la implementación del modelo en un sistema fragmentado. Los elementos de mayor consenso identificados, fueron: mayor disponibilidad de recursos económicos para garantizar la infraestructura y los recursos humanos suficientes y cualificados, con la figura del profesional de enfermería gestor de casos, como un recurso costo efectivo para el sistema de salud; implementación de rutas de atención integrales; sistemas de información que faciliten el tránsito de los pacientes entre prestadores y aseguradores, igual que los niveles de atención; vinculación de redes de apoyo, como asociaciones de pacientes y cuidadores que potencien la autogestión; $y$, priorizar la población en territorios rurales y áreas dispersas, por encontrarse en mayor condición de inequidad frente al acceso a los servicios.

- Por esto, aunque es un modelo nuevo en Colombia, se recomienda continuar el trabajo intersectorial con la participación de los diferentes actores, a fin de estudiar los beneficios y barreras para la implementación del modelo; del mismo modo, se aconseja el apoyar propuestas de investigación que muestren evidencias sólidas en diferentes ámbitos coordinados, facilitando la toma de decisiones frente al mejoramiento y la adaptación de la atención en salud, para la población crónica compleja a nivel nacional.

\section{Financiación}

Este producto hace parte del proyecto "Efectividad de un modelo de gestión de casos para la provisión integral de servicios de salud a pacientes pluripatológicos" financiado por el Departamento Administrativo de Ciencia, Tecnología e Innovación de Colombia (Colciencias), a través de la convocatoria 777 para proyectos de Ciencia, Tecnología e Innovación en Salud - 2017, número de contrato 848-2017. 
Revista

CienciaveCuidado

Scientific Journal of Nursing
Sandra Milena Hernández-Zambrano, Sonia Patricia Carreño-Moreno, Ruth Alexandra Castiblanco-Montañez, Lorena Mesa-Melgarejo, Lorena Chaparro-Díaz, Ana Julia Carrillo-Algarra, Elena Gonzalo-Jiménez

\section{Referencias Bibliográficas}

1. Organización Mundial de la Salud. Enfermedades no transmisibles [sede Web]. 2018 [Consultado 22 mayo 2020]. Disponible en: https://www.who.int/es/news-room/fact-sheets/detail/noncommunicable-diseases

2. NICE guideline. Multimorbidity: clinical assessment and management. London: National Institute for Health and Care Excellence. [Internet]. 2016 [Consultado 22 mayo 2020]. Disponible en: https://www.nice.org.uk/guidance/ng56

3. Junta de Andalucía. Atención a Pacientes Pluripatológicos. Sevilla: Consejería de Salud. [Internet] 2018 [Consultado 22 mayo 2020]. Disponible en: https:/www.juntadeandalucia.es/export/drupaljda/salud 5af1956d9925c atencion_pacientes_pluripatologicos_2018.pdf

4. Morales-Asencio JM, Martin-Santos FJ, Kaknani S, Morilla-Herrera JC, Cuevas Fernández-Gallego M, García-Mayor S, et al. Living with chronicity and complexity: Lessons for redesigning case management from patients' life stories - A qualitative study. J Eval Clin Pract [Internet]. 2016 [Consultado 22 mayo 2020]; 22(1):122-32. Disponible en: https://www.onlinelibrary.wiley.com/doi/abs/10.1111/jep.12300

5. Rodríguez-García J, Peñaloza-Quintero RE, Amaya-Lara JL, Amaya-Lara JL. Estimación de la carga global de enfermedad en Colombia 2012: nuevos aspectos metodológicos. Rev Salud Pública [Internet]. 2017 Mar 1 [Consultado 6 diciembre 2018];19(2):235-40. Disponible en: https://revistas.unal.edu.co/index.php/revsaludpublica/ article/view/66179

6. Londoño J. La carga de la enfermedad: un nuevo indicador en el campo de la salud pública. Fac Nac Salud Pública [Internet]. 2012 [Consultado 6 diciembre 2018];13(2):24-32. Disponible en: http://bibliotecadigital.udea. edu.co/bitstream/10495/4528/1/LondonoJuan_1996_CargaEnfermedadIndicador.pdf

7. Alfonso-Sierra E, Arcila Carabalí A, Bonilla Torres J, Latorre Castro ML, Porras Ramírez A, Urquijo Velásquez L. Situación de multimorbilidad en Colombia, 2012-2016. [Internet]. Colombia: Ministerio de Salud y Protección Social; 2018. [Consultado 6 diciembre 2018]. Disponible en: https://www.minsalud.gov.co/Ministerio/ DSector/Paginas/estudios-sectoriales.aspx

8. Barrera-Ortiz L, Carrillo-González GM, Chaparro-Díaz L, Sánchez-Herrera B. Modelo para abordar la carga del cuidado de la enfermedad crónica en Colombia. Orinoquía [Internet]. 2015 [Consultado 2 febrero 2019];19(1):89-99.

9. Duran-Badillo T, Salazar-González BC, Cruz-Quevedo JE, Sánchez-Alejo EJ, Gutiérrez-Sánchez G, Hernández-Cortés PL. Sensory and cognitive functions, gait ability and functionality of older adults. Rev. Latino-Am. Enfermagem [Internet]. 2020 [Consultado 15 octubre 2020];28. Disponible en: https://doi.org/10.1590/1518$\underline{8345.3499 .3282 .}$

10. Makovski TT, Schmitz S, Zeegers MP, Stranges S, van den Akker M. Multimorbidity and quality of life: systematic literature review and meta-analysis. Ageing Res Rev [Internet]. 2019 [Consultado 02 febrero 2020]. Disponible en: https://pubmed.ncbi.nlm.nih.gov/31048032/

11. Torres-Pinto X, Carreño-Moreno S, Chaparro-Diaz L. Factores que influencian la habilidad y sobrecarga del cuidador familiar del enfermo crónico. Rev la Univ. Ind Santander Salud [Internet]. 2017 [Consultado 27 junio 2017];49(2):330-8. Disponible en: http://revistas.uis.edu.co/index.php/revistasaluduis/article/view/6359/6594

12. Cabada E, Castillo VAM. Prevalencia del síndrome de sobrecarga y sintomatología ansiosa depresiva en el cuidador del adulto mayor. Psicol y Salud [Internet]. 2017 Feb [Consultado 02 febrero 2020];27(1):53-9. Disponible en: https://psicologiaysalud.uv.mx/index.php/psicysalud/article/download/2436/4287 
13. Darling-Izquierdo M, Manjarrés-Figueroa K, Nieves-Blanco W, Pacheco-Mercado B, Pérez-Mendivil J, Romero-Massa E. Nivel de sobrecarga y características del cuidado en cuidadores de personas con trastornos mentales, Cartagena [Tesis en internet]. Cartagena: Universidad de Cartagena; 2016. Disponible en: https://repositorio. unicartagena.edu.co/bitstream/handle/11227/3103/final-entregado-SUSTENTACION.pdf?sequence=1\&isAllowed $=\mathrm{y}$

14. Gonzalo Jiménez E (Coord.). Modelo de gestión de casos del servicio andaluz de salud. Guía de reorientación de las prácticas profesionales de la gestión de casos en el Servicio Andaluz de Salud. [Internet]. Sevilla: Servicio Andaluz de Salud. Consejería de Salud. Junta de Andalucía; 2017. Disponible en: http://www.juntadeandalucia.es/servicioandaluzdesalud/contenidos/publicaciones/datos/686/pdf/mgc_modelo_gestion_casos.pdf

15. Pimouguet C, Lavaud T, Dartigues JF, Helmer C. Dementia case management effectiveness on health care costs and resource utilization: a systematic review of randomized controlled trials. J Nutr Health Aging [Internet]. 2010 Oct [Consultado el 6 de diciembre de 2018];14(8):669-76. Disponible en: http://www.ncbi.nlm.nih.gov/ pubmed/20922344

16. Everett B, Castel LD, McGinnis M, Beresky A, Cane RC, Cooper T, et al. Economic and Clinical Outcomes Resulting From the Stage 4 Chronic Kidney Disease Case Management Quality Improvement Initiative. Prof Case Manag [Internet]. 2017 [Consultado 7 diciembre 2018];22(6):291-8. Disponible en: http://www.ncbi.nlm. nih.gov/pubmed/28902768

17. Hudon C, Chouinard M-C, Aubrey-Bassler K, Muhajarine N, Burge F, Pluye P, et al. Case management in primary care among frequent users of healthcare services with chronic conditions: protocol of a realist synthesis. BMJ Open [Internet]. 2017 [Consultado 7 diciembre 2018];7(9): e017701. Disponible en: http://www.ncbi.nlm. nih.gov/pubmed/28871027

18. García Mayor S. Effectiveness of advanced practice nursing interventions in older people and their description through the nursing interventions classification. a systematic review and qualitative study [Tesis en Internet]. Málaga: Universidad de Málaga; 2014. Disponible en: https://dialnet.unirioja.es/servlet/tesis?codigo=45979

19. Coderch J, Perez-Berruezo X, Sánchez-Pérez I, Sánchez E, Ibern P, Pérez M, et al. Evaluación de la efectividad de un programa de atención integrada y proactiva a pacientes crónicos complejos. Gac.Sanit [Internet]. 2018 [Consultado el 30 de abril de 2020]; 32(1): 18- 26. Disponible en: https://doi.org/10.1016/j.gaceta.2016.07.014

20. Varela-Ruiz M, Díaz-Bravo L, García-Duran R. Descripción y usos del método Delphi en investigaciones del área de la salud. Inv. Ed Med [Internet]. 2012 [Consultado 30 abril 2020]; 1(2): 90-95. Disponible en: http://riem. facmed.unam.mx/sites/all/archivos/V1Num02/07 MI DESCRIPCION Y USOS.PDF

21. Hernández-Zambrano SM, Mesa-Melgarejo L, Carrillo-Algarra AJ, Castiblanco-Montañez RA, Chaparro-Diaz L, Carreño-Moreno SP, et al. Effectiveness of a case management model for the comprehensive provision of health services to multi-pathological people. J Adv Nurs [Internet] 2019; [Consultado 20 abril 2020] 75: 665675. Disponible en: https://doi.org/10.1111/jan.13892

22. Miguélez-Chamorro A, Casado-Mora MI, Company-Sancho MC, Balboa-Blanco E, Font-Oliver MA, Román-Medina I. Advanced practice in case management: An essential element in the new complex chronicity care model. Enferm Clin [Internet]. 2019 [Consultado 20 abril 2020]; 29(2):99-106. Disponible en: https://doi. org/10.1016/j.enfcli.2018.08.005

23. Morilla JC, Morales-Asencio JM, Kaknani S, García Mayor S. Complex chronic care situations and socio-health coordination. Enferm Clín [Internet].2016 [Consultado 20 abril 2020];26(1): 55-60. Disponible en: https://doi. org/10.1016/j.enfcli.2015.08.001

24. Woo BFY, Lee JXY, Tam WWS. The impact of the advanced practice nursing role on quality of care, clinical outcomes, patient satisfaction, and cost in the emergency and critical care settings: A systematic review. Hum 


\section{Cienciäocuidado}

Scientific Journal of Nursing
Sandra Milena Hernández-Zambrano, Sonia Patricia Carreño-Moreno, Ruth Alexandra Castiblanco-Montañez, Lorena Mesa-Melgarejo, Lorena Chaparro-Díaz, Ana Julia Carrillo-Algarra, Elena Gonzalo-Jiménez

Resour Health [Internet].2017 [Consultado 25 abril 2020];15(1): Disponible en: https://doi: 10.1186 / s12960-0170237-9.

25. Duarte Clíments G, Rodríguez Álvarez C, Sierra López A, Rodríguez Gómez JÁ, Aguirre JA, Sánchez Gómez MB. Evaluación del Modelo de Gestión de Casos en Atención Primaria mediante enfermeras comunitarias de enlace. Ene. [Internet]. 2019 [Consultado 25 abril 2020];13(3). Disponible en: http://scielo.isciii.es/scielo. php?script $=$ sci arttext\&pid=S1988-348X2019000300006\&lng $=$ es

26. Naganathan G, Kuluski K, Gill A, Jaakkimainen L, Upshur R, Wodchis WP. Perceived value of support for older adults coping with multi-morbidity: Patient, informal caregiver and family physician perspectives. Ageing Soc[Internet] 2016 [Consultado 25 abril 2020];36(9):1891-914. Disponible en: https://oi.org/10.1017/ $\underline{\mathrm{S} 0144686 \mathrm{X} 15000768}$

27. Melo BG, Vargas Hernández Y, Carrillo GM, Alarcón Trujillo DK. Effect of the programme Plan de egreso on chronically ill patients and their family caregivers. Enferm Clíni [Internet]. 2018 [Consulado 25 abril 2020];28(1):36-43. Disponible en: http://dx.doi.org/10.1016/j.enfcle.2017.09.003

28. Kuipers, S.J., Nieboer, A.P. \& Cramm, J.M. Views of patients with multi-morbidity on what is important for patient-centered care in the primary care setting. BMC Fam Pract [Internet]. 2020 [Consultado 25 abril 2020]; 21(71) Disponible en: https://doi.org/10.1186/s12875-020-01144-7

29. Dalmolin Indiara S, Heidemann IT. Integrative and complementary practices in Primary Care: unveiling health promotion. Rev. Latino-Am. Enfermagem [Internet]. 2020 [Consultado 20 abril 2020];28. Disponible en: https:// doi.org/10.1590/1518-8345.3162.3277.

30. Joo JY y Liu MF. Case management effectiveness for managing chronic illnesses in Korea: a systematic review. Int Nurs Rev [Internet]. 2019 Mar [Consultado 20 abril 2020];66(1):30-42. Disponible en: https://pubmed.ncbi. nlm.nih.gov/29956825/

31. Morales-Asencio JM, Cuevas-Fernández-Gallego M, Morilla-Herrera JC, Martín-Santos FJ, Silvano Arranz A, Batres Sicilia JP, et al. Characteristics of the provision of case management services in the community setting in Andalusia based on the RANGECOM registry. Enferm Clin [Internet]. 2019 [Consultado 25 abril 2020]; 29(2):67-73. Disponible en: https://doi.org/10.1016/j.enfcli.2018.12.009

32. Rijken M, van der Heide I. Identifying subgroups of persons with multimorbidity based on their needs for care and support. BMC Fam Pract [Internet]. 2019 [Consultado 25 abril 2020];20(179). Disponible en: https://doi. org/10.1186/s12875-019-1069-6

33. Stokes J, Panagioti M, Alam R, Checkland K, Cheraghi-Sohi S, Bower P. Effectiveness of Case Management for "At Risk" Patients in Primary Care: A Systematic Review and Meta-Analysis. PLoS One [Internet]. 2015 [Consultado 20 abril 2020];10(7). Disponible en: https://pubmed.ncbi.nlm.nih.gov/26186598/

34. Ministerio de salud y Protección Social, Cendex, U. Javeriana. [Internet] Estudio de disponibilidad y distribución de la oferta de médicos especialistas, en servicios de alta y mediana complejidad en Colombia. [Consultado 02 mayo 2020]. Disponible en: https://www.minsalud.gov.co/salud/Documents/Observatorio $\% 20$ Talento $\% 20$ Humano $\% 20$ en $\% 20$ Salud/DisponibilidadDistribuci $\%$ C3\%B3nMdEspecialistasCendex.pdf 